www.mdpi.com/journal/remotesensing

Article

\title{
Fraunhofer Lidar Prototype in the Green Spectral Region for Atmospheric Boundary Layer Observations
}

\author{
Songhua Wu *, Xiaoquan Song and Bingyi Liu \\ Ocean Remote Sensing Institute, Ocean University of China, 238 Songling Road, Qingdao 266100, \\ China; E-Mails: songxq@ouc.edu.cn (X.S.); bingyiliu@ouc.edu.cn (B.L.) \\ * Author to whom correspondence should be addressed; E-Mail: wush@ouc.edu.cn; \\ Tel.: +86-532-6678-2573.
}

Received: 8 October 2013; in revised form: 27 October 2013 / Accepted: 13 November 2013 / Published: 18 November 2013

\begin{abstract}
A lidar detects atmospheric parameters by transmitting laser pulse to the atmosphere and receiving the backscattering signals from molecules and aerosol particles. Because of the small backscattering cross section, a lidar usually uses the high sensitive photomultiplier and avalanche photodiode as detector and uses photon counting technology for collection of weak backscatter signals. Photon Counting enables the capturing of extremely weak lidar return from long distance, throughout dark background, by a long time accumulation. Because of the strong solar background, the signal-to-noise ratio of lidar during daytime could be greatly restricted, especially for the lidar operating at visible wavelengths where solar background is prominent. Narrow band-pass filters must therefore be installed in order to isolate solar background noise at wavelengths close to that of the lidar receiving channel, whereas the background light in superposition with signal spectrum, limits an effective margin for signal-to-noise ratio (SNR) improvement. This work describes a lidar prototype operating at the Fraunhofer lines, the invisible band of solar spectrum, to achieve photon counting under intense solar background. The photon counting lidar prototype in Fraunhofer lines devised was used to observe the atmospheric boundary layer. The SNR was improved 2-3 times by operating the lidar at the wavelength in solar dark lines. The aerosol extinctions illustrate the vertical structures of aerosol in the atmospheric boundary over Qingdao suburban during summer 2011.
\end{abstract}

Keywords: lidar; Fraunhofer lines; photon counting; signal-to-noise ratio; aerosol extinction coefficient 


\section{Introduction}

Lidar (light detection and ranging) or laser radar detects atmospheric parameters by transmitting laser pulse to the atmosphere and receiving the backscattering signals from molecules and aerosol particles, described by Rayleigh scattering and Mie scattering [1]. Mie scattering is generally the most efficient of these scattering processes with cross-sections of the order $10^{-26}-10^{-8} \mathrm{~cm}^{2} /$ particle, whereas Rayleigh cross-sections are in the region of $4.04 \times 10^{-27}-7.24 \times 10^{-27} \mathrm{~cm}^{2} /$ molecule in the wavelength region from $490 \mathrm{~nm}$ to $565 \mathrm{~nm}$ [2]. This Rayleigh cross-section follows a $\lambda^{-4}$ relation and, hence, shorter wavelengths exhibit greater scattering effects. Because of the extreme low backscattering cross section, lidar usually uses the high sensitive photon detector and photon counting technology to obtain the return signal from long distance by a long time accumulation [3-11]. Because of the strong solar background, the signal-to-noise ratio (SNR) of lidar during daytime could be greatly restricted, especially for the lidar operating at visible spectral region where solar background is prominent [7]. A large number of background rejection techniques have been used to improve the SNR such as narrow band interference filter, Fabry-Perot etalon and field-of-view restriction, whereas the solar noise in superposition with the signal spectrum limits an effective margin for SNR improvement.

The absorption process of solar radiation is well known as the solar Fraunhofer spectra due to certain atmospheric constituent in either the solar atmosphere or that of the earth, first documented and mapped by Joseph von Fraunhofer in 1812. Because of the absorption process, the solar light reaching the earth's surface exhibits certain "dark windows" rather than being a continuum of all wavelengths. Modern observations of solar spectrum can detect many thousands of lines. The wavelengths of the most notable lines for visible wavelengths $[12,13]$ are given in Figure 1. Fraunhofer lines produce strong absorptions and fingerprint information of sunlight. Therefore, those lines have been studied for free-space laser communications and passive optical sensing techniques [14,15]. A wavelength lying within a Fraunhofer line can carry optical communications with reduced interference from the solar background. The tunable source laser and the Fraunhofer filter were proposed to match the Doppler shifts of the source and background [14]. A free-space Quantum key distribution system for Earth-space data link was described that lies within an H-alpha Fraunhofer line at $656.28 \mathrm{~nm}$, to carry the signal with reduced interference from sunlight [15]. Fraunhofer line radiometers were also suggested to identify certain materials of solar-stimulated luminescence [16,17]. A space mission called FLEX (Fluorescence Explorer) probe terrestrial vegetation by solar induced fluorescence that is detectable within the Fraunhofer lines in the red and blue-UV region [18]. With regards to laser remote sensing, resonance fluorescence techniques have evolved to measure mesospheric metals such as $\mathrm{Na}$, $\mathrm{K}, \mathrm{Fe}, \mathrm{Ca}$, and $\mathrm{Ca}^{+}$are capable of measuring mesospheric metals densities in the middle atmosphere region 80-105 km [19-24]. The thermal broadening and Doppler frequency shift of the fluorescence spectrum of such metals have also been used to retrieve temperature and wind structures throughout the mesospheric region. These middle-atmosphere lidars spontaneously exhibit good signal-to-noise ratio during the daytime because the resonance fluorescence wavelengths of such metals are consistent with the strong Fraunhofer lines by the same metal atoms or ions in the solar atmosphere [25]. This work describes a photon counting lidar prototype operating at the Fraunhofer lines in the green spectral region, the invisible spectral region of the solar spectrum, to achieve photon counting under intense solar background. The first criterion to select the dark lines is that the line has a strong absorption peak 
to minimize solar noise at the signal wavelength. The second criterion is that the line has broad linewidth to facilitate tuning of both the laser transmitter and optical filter. The last consideration is that the dark line is co-inside with some atomic or molecular absorption filter as potential high spectral resolution receiver. Based on the above criteria, it was found that three such lines exist, at $516.7327 \mathrm{~nm}, 517.2698 \mathrm{~nm}$ and $518.3619 \mathrm{~nm}$, by the Magnesium atom in the solar chromospheres. The broadest linewidth of those is approximately $0.2 \mathrm{~nm}$ wide, slightly broader than that of tunable laser we have, $0.17 \mathrm{~nm}$. Thus, optimization of the receiver optics such as high resolution monochromator/spectrograph is investigated to cover the margin to sufficiently minimize the solar noise such that equivalent photon counting efficiency in daytime can be obtained. The laser transmitter is an optical parametric oscillator pumped by $355 \mathrm{~nm}$ $\mathrm{Nd}$ :YAG (neodymium-doped yttrium aluminum garnet) laser and is tuned to the selected dark lines by which nearly $90 \%$ of solar radiation is absorbed. The prototype provides some theoretical references for optimized design of photon counting lidar in the visible spectral region with comparable SNR at night, which may contribute to the research on diurnal variation of the atmosphere and to the operational observation of lidar. The ongoing procedures included the application of atomic vapor filter as the receiver taking maximum heritages of traditional HSRL for wind, temperature and aerosol probing [6-10].

Figure 1. Fraunhofer lines for visible wavelengths [12].

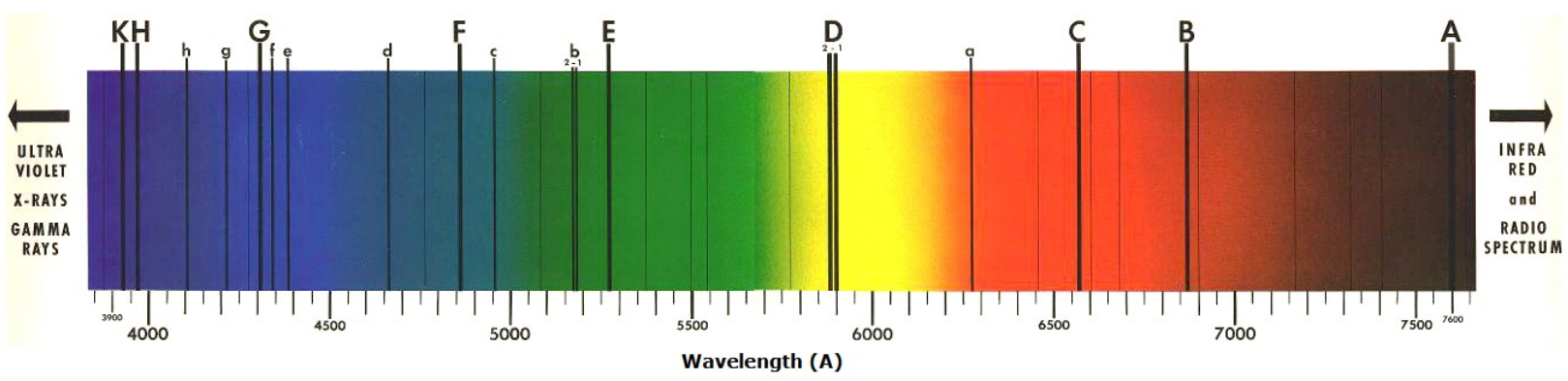

\section{Apparatus}

\subsection{Apparatus and Methodology}

The optical layout of the lidar prototype is shown in Figure 2. The laser used for this application is a tunable OPO (Optical Parametric Oscillator) laser pumped by the third harmonic of a Nd:YAG laser. It is found that the divergence of the laser output is too large to collect backscattered light. A pair of cylinder lens and a beam expander is installed in front of the OPO laser output to produce a circular beam pattern with a lower divergence. A 5 times beam expander follows the cylinder lens to reduce the beam divergence. The backscattering signal is collected by a Schmidt-Cassegrain telescope with 200 $\mathrm{mm}$ diameter and is coupled into the fiber bundles that contain 19 fibers, $200 \mu \mathrm{m}$ diameter each, arranged in a line pattern at the slit end, and round at the source end. The fiber guides the backscattering light into a $500 \mathrm{~mm}$ focal length monochromator/spectrograph that has adjustable spectral resolution from $0.02 \mathrm{~nm}$ to $5 \mathrm{~nm}$. The photons are detected with a photomultiplier in the exit slit of the monochomater. The photoelectric current is digitized by photon counting technique with a preamplifier. The dashed diagram shows an optional receiver assembly as high spectral resolution 
receiver consisting of two channels. One of two channels is a molecular vapor filter and the other is the reference channel. The system specification is listed in Table 1.

Figure 2. Optical layout of the Fraunhofer lidar prototype.

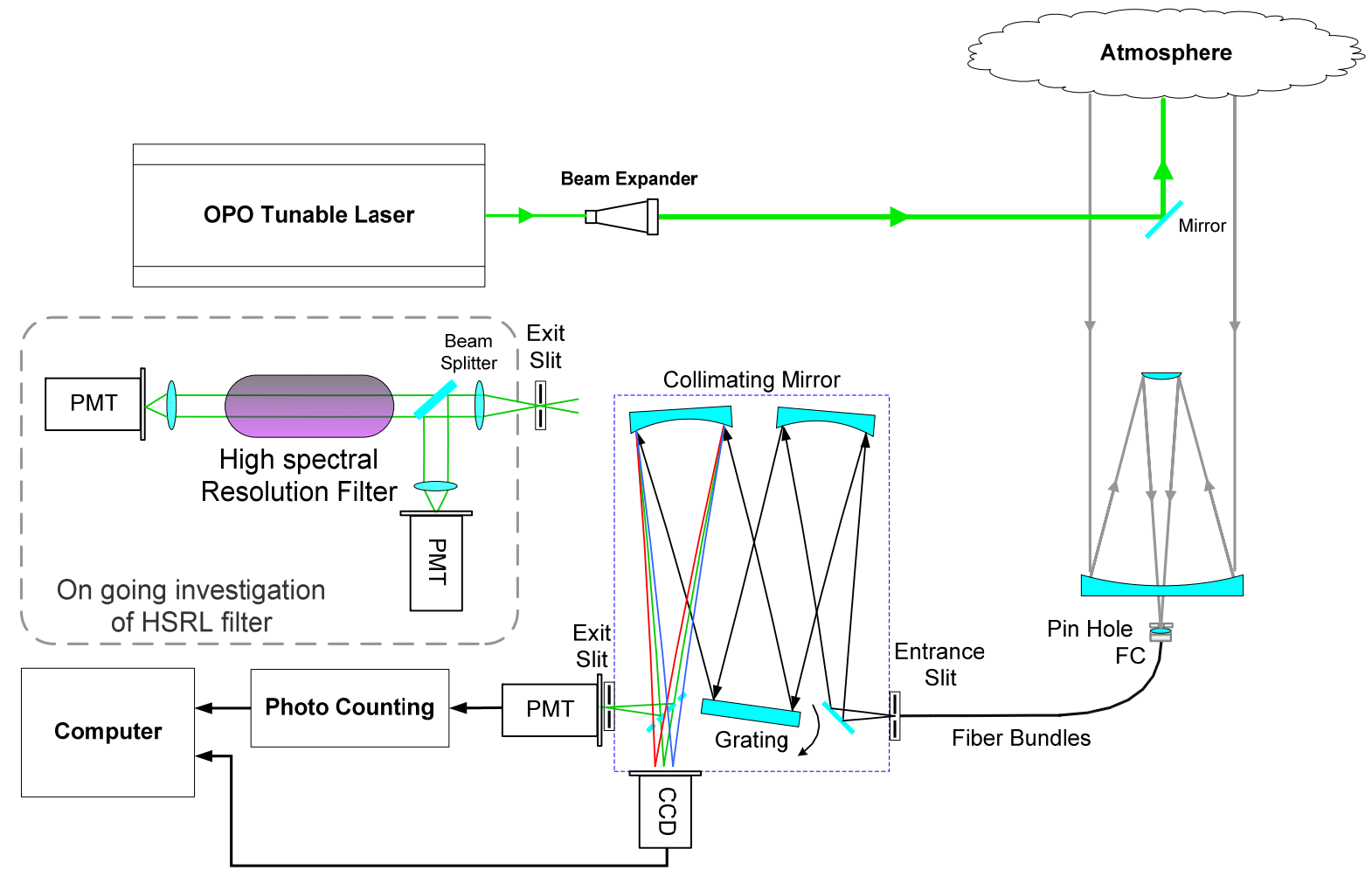

Table 1. Specifications of the Fraunhofer Lidar.

\begin{tabular}{|c|c|}
\hline \multicolumn{2}{|c|}{ Pump Laser Specifications } \\
\hline Pump Laser & Nd:YAG, Q-Switched flash lamp pumped \\
\hline Pump wavelength & $355 \mathrm{~nm}$ \\
\hline Pump pulse energy & $180 \mathrm{~mJ}$ \\
\hline Pulse repetition rate & $10 \mathrm{~Hz}$ \\
\hline Pulse width & $5 \mathrm{~ns}$ \\
\hline \multicolumn{2}{|c|}{ OPO Parameters } \\
\hline Wavelength Tuning Range & $410-2,400 \mathrm{~nm}$ \\
\hline Peak OPO Energy & $30 \mathrm{~mJ}$ \\
\hline Spectral Linewidth & $4-7 \mathrm{~cm}^{-1}(0.1-0.2 \mathrm{~nm})$ \\
\hline Beam Divergence & Vertical: $<2 \mathrm{mrad}$ Horizontal: $<10 \mathrm{mrad}$ \\
\hline Polarization & Signal: Horizontal, Idler: Vertical Linear Polarization \\
\hline \multicolumn{2}{|r|}{ Optical Filter } \\
\hline Optical Design & Imaging Czerny-Turner \\
\hline Grating & 1,200 grooves $/ \mathrm{mm}$ \\
\hline Dispersion & $1.7 \mathrm{~nm} / \mathrm{mm}$ \\
\hline Resolution & $0.02 \mathrm{~nm}$ at $435.8 \mathrm{~nm}$ \\
\hline Repeatability & $\pm 0.05 \mathrm{~nm}$ \\
\hline Drive Step Size & $0.0025 \mathrm{~nm}$ \\
\hline Slits & $10 \mu \mathrm{m}$ to $3 \mathrm{~mm}$ adjustable \\
\hline
\end{tabular}


Table 1. Cont.

\begin{tabular}{cc}
\hline & Data Acquisition \\
\hline Photomultiplier & Hamamatsu R7400U-20 \\
Cathode radiant sensitivity & $>70 \mathrm{~mA} / \mathrm{W}$ \\
Maximum Photon Counting Rate & $250 \mathrm{MHz}$ \\
Sampling resolution & $3.75 \mathrm{~m}$ \\
\hline
\end{tabular}

\subsection{Methodology}

The solar spectra background is measured by the spectrograph and a CCD camera in order to confirm the presence of the selected lines. The spectrograph is calibrated by a Mercury lamp, prior to the background investigation. The sky light is collected by the telescope and coupled into a spectrograph, chosen for its high resolution with a diffraction grating of 1,200 grooves per mm and a focal length of $500 \mathrm{~mm}$. The dispersion is $1.7 \mathrm{~nm} / \mathrm{mm}$ and the pixel width of the CCD is $12.5 \mu \mathrm{m}$, so as, that the "snap shot" of solar background has a spectral resolution of $0.0213 \mathrm{~nm}$. Figure 3a shows the solar spectrum between $515 \mathrm{~nm}$ to $520 \mathrm{~nm}$ measured by the spectrum channel of lidar on May 4, 2011. The solar spectrum within same region [13] is illustrated in Figure $3 \mathrm{~b}$ for reference. The solar spectrum clearly indicates the solar Fraunhofer lines at $516.4521 \mathrm{~nm}, 517.0414 \mathrm{~nm}$ and $518.2386 \mathrm{~nm}$. The frequency shift of the observed lines with respect to the literature is supposed to be affected synthetically by the imperfect calibration for the spectrometer and the effect of local Doppler shift due to the Earth revolution about the sun [14]. More hyperfine structure from various absorption and scatters in both earth and solar atmosphere are also observed in the figure. Figure 3 shows the dark lines at $517.0414 \mathrm{~nm}$ and $518.2386 \mathrm{~nm}$ with a minimum of around 10000 counts and a width of $0.2 \mathrm{~nm}$ which is slightly wider than the OPO laser linewidth at that wavelength $(0.18 \mathrm{~nm}$ and $0.16 \mathrm{~nm}$ respectively) (Figure 4) and therefore these two lines are chosen. The spectral separation of both wavelengths is $1.1972 \mathrm{~nm}$ according to the measurement.

Figure 3. (a) The solar spectra background measured by lidar; (b) the solar spectrum provided by Kurucz 2010 [13].

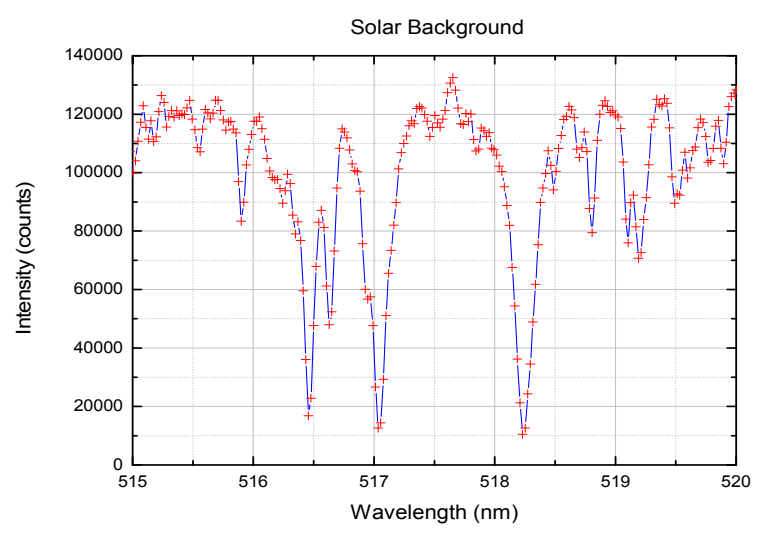

(a)

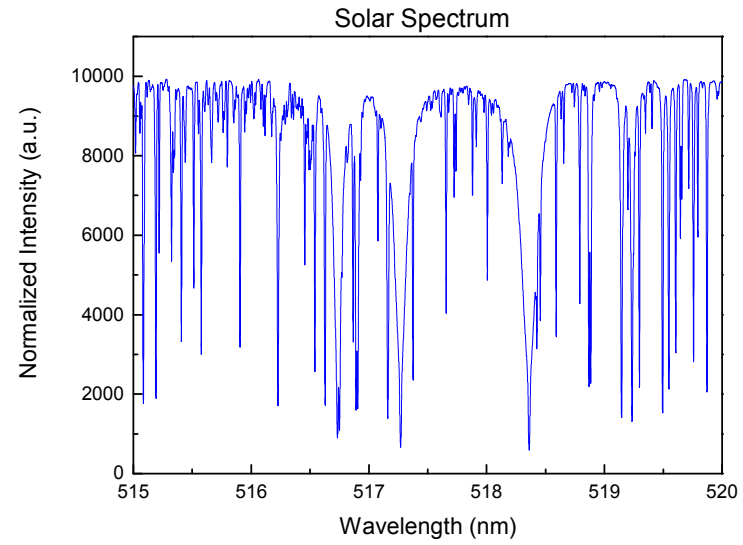

(b) 
Optimization of spectral matching is performed by the combination of collimating slits, placed at both the entry and exit to the spectrometer, and the best resolution and signal-to-noise ratio results were achieved with both slits set at $100 \mu \mathrm{m}$. In this case, a photomultiplier tube Hamamatsu R7400U-20 was placed at the exit slit of the spectrometer, and the signal output fed into a photon counter. Tuning of the OPO laser was achieved by tuning the grating to the minimum of the solar dark line, scanning the OPO laser past this wavelength region, and measuring the returned signal.

Figure 4. Linewidth of the OPO laser measured by the spectrograph.

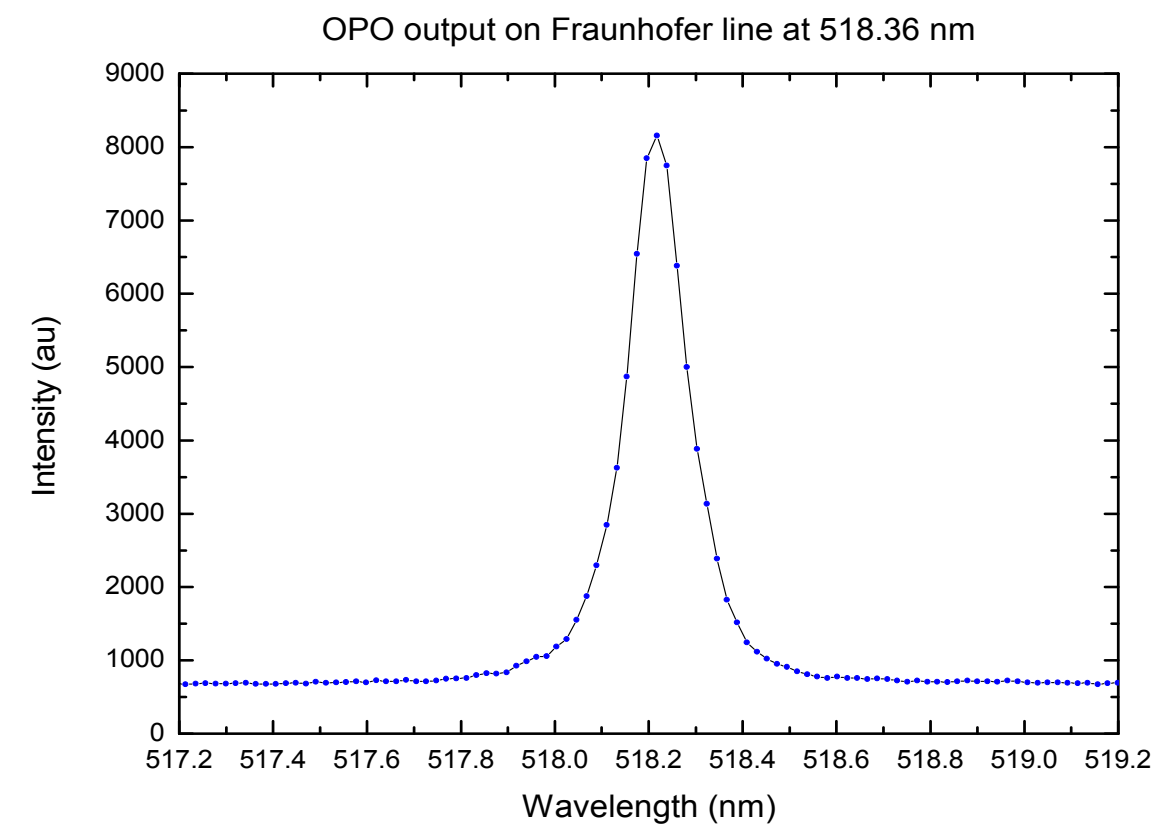

\section{Solar Background Analysis for Selected Fraunhofer Line}

In order to precisely match the laser wavelength and the Fraunhofer lines, the spectral channel using a CCD camera was continuously monitoring the skylight background and the laser backscatters when the laser transmitter was tuning to the minimum of the solar dark line. Figure 5 shows the superposition of the solar spectrum and the laser signal which fills the absorption dip of $\mathrm{Mg}$ Fraunhofer line at $518.2386 \mathrm{~nm}$. The energy of the laser was attenuated for better illustration.

The background level is evaluated for the on- and off-line wavelength of the Fraunhofer line. The decrease of the background from day to night is shown in Figure 6. The measurements for on- and off-line wavelength were alternately carried out by the one lidar system with the same configurations. It is assumed that all the circumstance factors like sun-zenith angle have the same impact on these very close wavelengths. The maximum of the background was observed at 15:00 pm because of the telescope pointing approximately at the sun. It can be seen that the difference in on- and off-line wavelength for the solar background is of the order of $70 \%$, which is less than the theoretical estimation. This is mostly due to the insufficient resolution of the spectrograph. Solar noises whose wavelength is not in a superposition with laser signal are also important in SNR. As we use a rotating grating system with the blocking ratio of OD (Optical Density) 6 (corresponding to $10^{-6}$ ). In order to compress the solar background and the stray light in the grating system, an additional band-pass filter 
having a linewidth of $10 \mathrm{~nm}$ and the blocking ratio of OD 5 is used between the telescope and the input port of the receiving fiber. Thus the total blocking ratio is about OD 11 that is sufficient for the solar background compression out of the spectral region of the Fraunhofer line. In normal case, solar background light comes into the photomultiplier through the filter because of the low blocking ratio of the spectrograph. In this case, the narrow band Fraunhofer absorption line is this not so effective in decreasing background solar background signal.

Figure 5. Laser transmitter spectrum and sky background with Fraunhofer lines structure.

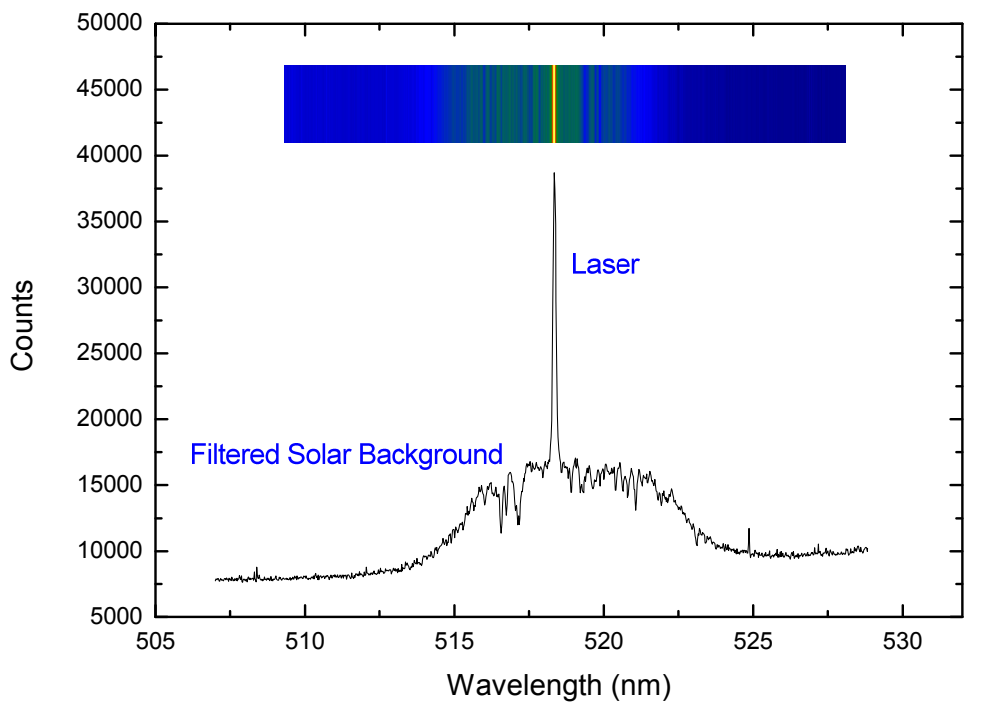

Figure 6. Variation of the on-line and off-line wavelength from the sky background from 07:00 to 11:00 UTC (15:00-19:00 LST)

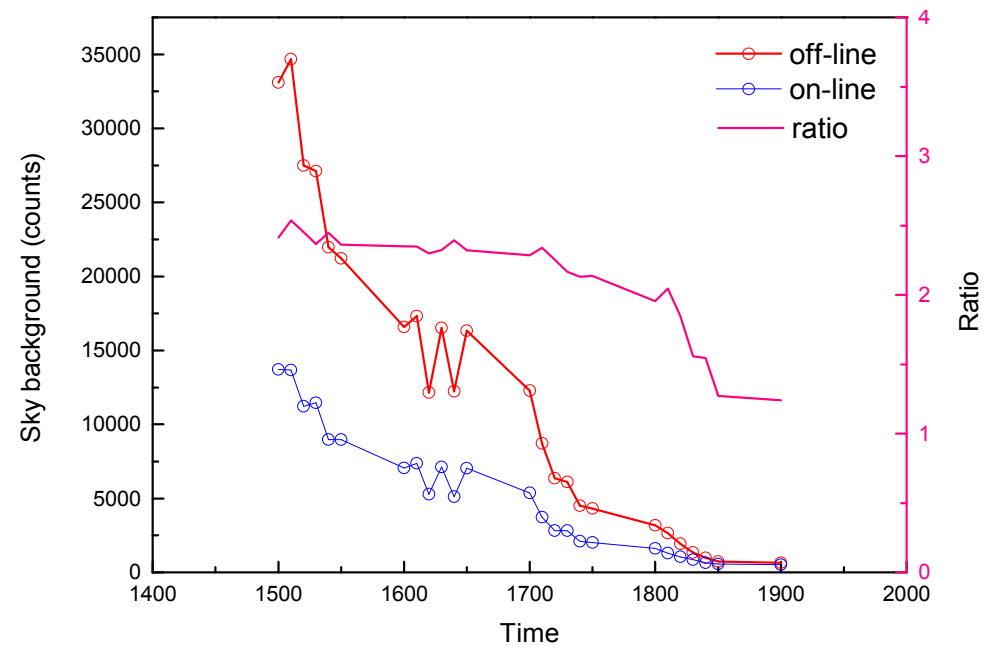

The output laser is tuned to $518.2386 \mathrm{~nm}$ (on-line) and $518.65 \mathrm{~nm}$ (off-line), respectively. Then we analyses the lidar SNR according to the lidar returned signal. The SNR is defined as Equation (1):

$$
S N R=\frac{N_{s}}{\sqrt{N_{s}+N_{b}+N_{e}}}
$$


where $\mathrm{N}_{\mathrm{s}}$ is laser backscattering signal, $\mathrm{N}_{\mathrm{b}}$ is the noise introduced by photons of the sky background, and $\mathrm{N}_{\mathrm{e}}$ is the noise from the dark current and readout electronics. $\mathrm{N}_{\mathrm{b}}$ and $\mathrm{N}_{\mathrm{e}}$ are estimated by the square of the standard deviation of the lidar return signal from the far distance where the background noise and electronics noise dominate. Figure $7 \mathrm{a}$ shows the SNR of the returned signal on-line and off-line measurements at 07:00 UTC (15:00 LST). The ratio of on-line SNR to off-line SNR is calculated according to Equation (2). The SNR on-line is obviously higher than the SNR off-line as shown in Figure $7 \mathrm{~b}$.

$$
\text { Ratio }=S N R_{\text {on-line }} / S N R_{\text {off }- \text { line }}
$$

For further contrast, Figure $8 \mathrm{a}$ shows the SNR of the returned signal on-line and off-line measurements at night for the same day while Figure $8 \mathrm{~b}$ shows the ratio between them. As Figure 8 presents, the SNR for the on-line and off-line wavelength are almost equivalent at night measurement. To draw a conclusion, it is very significant to tune the OPO output laser to the minimum of the solar dark line in order to improve the SNR of the Fraunhofer lidar, especially for daytime measurements.

Figure 7. (a) The SNR of on line and off line measurements; (b) The SNR difference between on-line and off-line measurements, at 07:00 UTC (daytime 15:00 LST), on 3 June 2011.

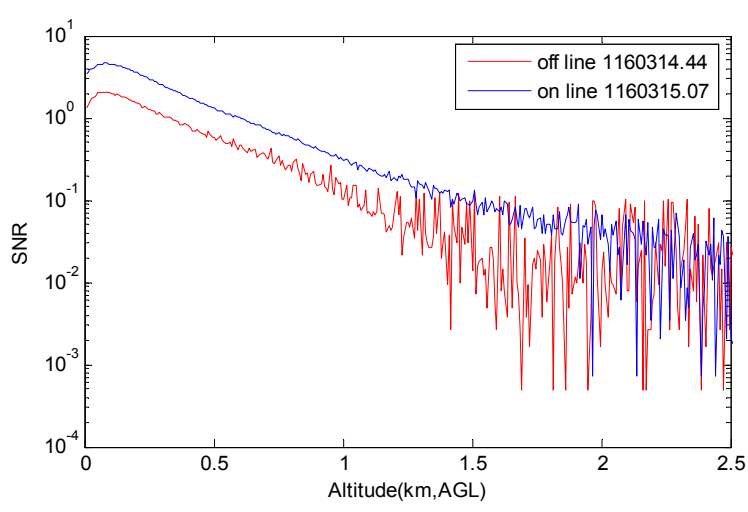

(a)

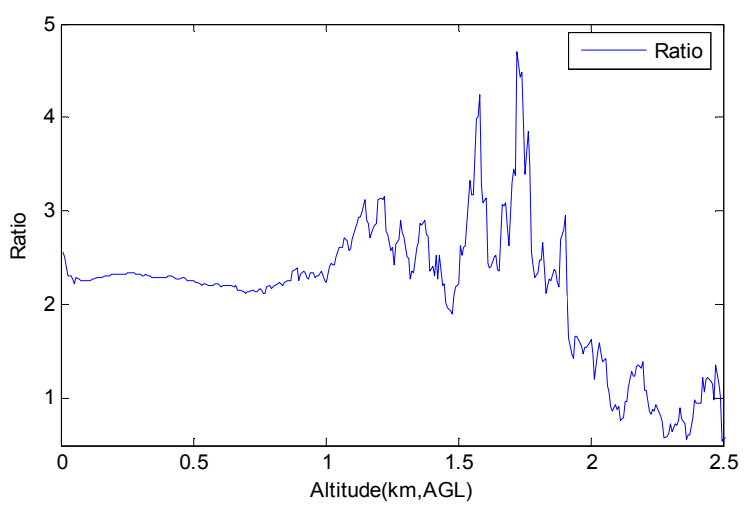

(b)

Figure 8. (a) The SNR of on-line and off-line measurements at night; (b) The SNR difference between on-line and off-line measurements at 12:00 UTC (night, 20:00 LST) on 3 June 2011.

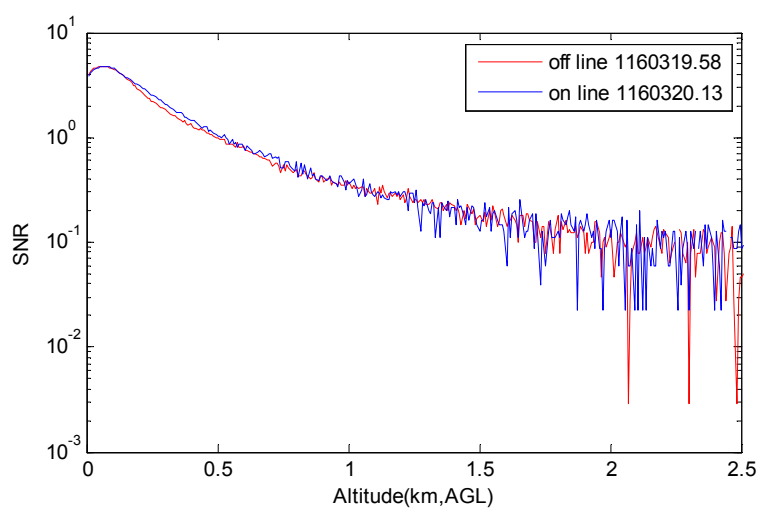

(a)

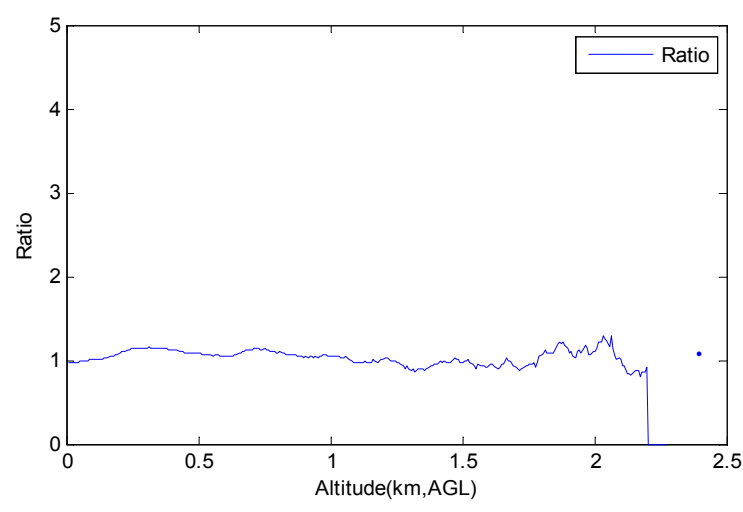

(b) 
The above lidar return signals were taken at clear and stable weather. In order to eliminate the pulse energy jitter and long term power drift of the transmitting laser, which is prominent for the OPO laser we used, an extra photomultiplier was adopted as reference to eliminate the variation of the laser power. The SNR of the lidar backscatter signals on 3 June 2011 at both noon and night are compared in Figure 9. The analysis shows that the SNR of the measurements in the daytime are almost equivalent to that of night measurements for SNR $>10$ decibels. The variation of the SNR between daytime and nighttime is less than \pm 1 decibels. For the far distance lidar return, night measurements have better SNR than the daytime measurement due to the lower background noise at night (for SNR < 10 decibels ).

Figure 9. SNR comparison between day and night measurements.

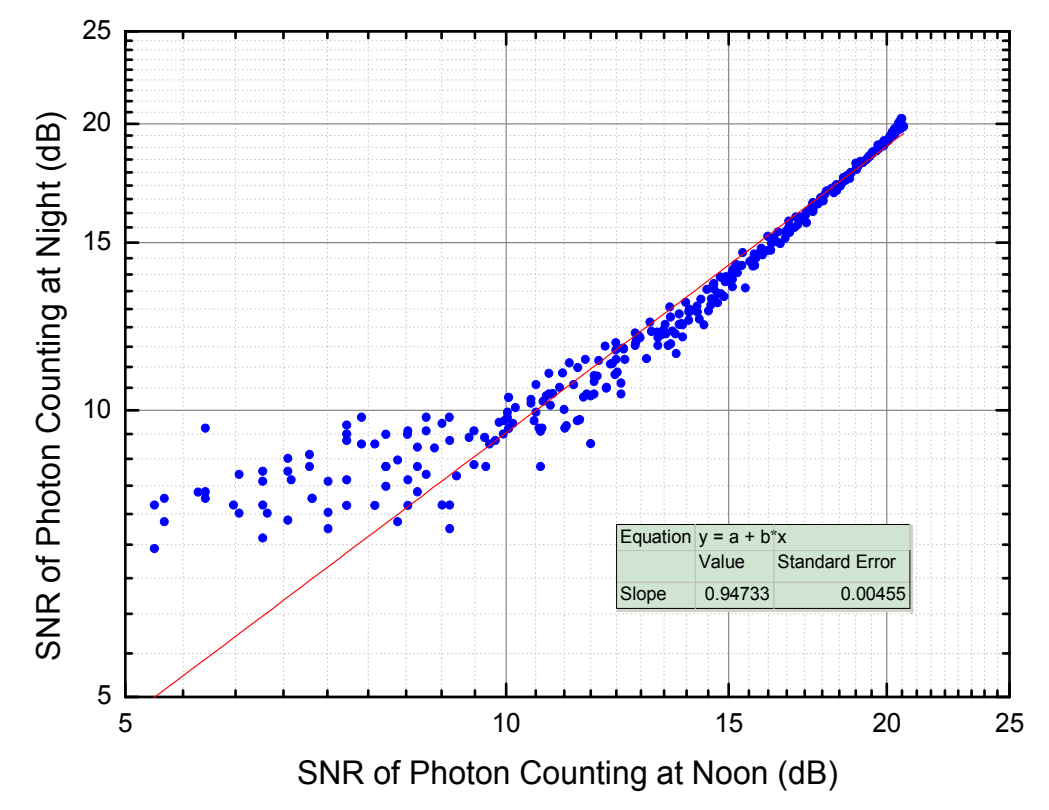

\section{Atmospheric Boundary Layer Observations}

According to the above analysis, the prototype lidar exhibits the advantage of photon counting at wavelengths corresponding to Fraunhofer lines in the green spectral region with strong solar irradiance. Two measurement cases obtained at different meteorological and aerosol conditions, are presented to demonstrate the capability of the Fraunhofer lidar prototype for the atmospheric boundary layer (ABL) observation. The lidar was located at the Laoshan campus of the Ocean University of China $6 \mathrm{~km}$ away from the seashore and the elevation is $74 \mathrm{~m}$ above the mean sea level, where the longitude and latitude is $36.16^{\circ} \mathrm{N}$ and $120.49^{\circ} \mathrm{E}$ respectively. As the prototype lidar consists of only an elastic scattering channel, we adopt the well-known Fernald method [26] to retrieve the aerosol extinction coefficient in the boundary layer. It is requested to determine the reference altitude before calculating the aerosol extinction coefficient of a certain altitude, which is lower than the reference altitude. In this article, the reference altitude is determined at 2-3 km where the aerosol extinction coefficient of the altitude is $0.13 \mathrm{~km}^{-1}$. To solve the underconstrained lidar equation scince it is associated with two unknowns for one equation, we need to know an extinction-to-backscatter ratio, so-called "lidar ratio", to retrieve the aerosol extinction coefficient. Lidar ratio is related to the size, the 
shape, and the composition of the particles. The measurements were held in the summer and the sea breeze is a regular feature of the summer season. The lidar ratio for the marine aerosol is between 20 to $35 \mathrm{sr}$ [27]. The aerosol optical depths (AOD) can be used to retrieve an average lidar ratio [28]. For this study, the Moderate Resolution Imaging Spectroradiometer (MODIS) derived AOD was used for the case of boundary layer measurement in cloudless conditions and was used for the lidar ratio inversion.

The evolution of the atmospheric boundary layer under cloudless conditions during daytime is shown first (3 June 2011). In the second case (24 August 2011), the boundary layer with more variation (clouds on top of the boundary layer) is discussed and validated with the results of the space-borne lidar CALIOP onboard CALIPSO satellite.

Figure 10 presents the aerosol extinction coefficient profile above the ground level on June 3, 2011, for the lidar ratio of 20,30 and $35 \mathrm{sr}$, respectively. As shown in this figure, although it has slight difference when the altitude is lower than $1 \mathrm{~km}$, the tendency of these three profiles is consistent. A ratio of $30 \mathrm{sr}$ is used in this work.

In order to detect the vertical profile of the extinction coefficient and compare the detection performance between daytime and nighttime, the experiments were carried out when it was sunny and cloudless. The measurement was performed from 10:00 am to 20:00 pm when the weather conditions allowed from May to August. The 6 min averaged lidar profiles, comprising 3,600 shots acquired, were used for the extinction coefficient inversion.

For the first case, one scene of the boundary layer during clear sky condition is shown. Figure 11 presents the vertical extinction coefficient profiles for different times on 3 June 2011. As shown in the figure, the aerosol under $1.5 \mathrm{~km}$ increases slowly from 02:30 to 10:30 UTC (from 10:30 to 18:30 LST) and decreases from 08:31 to 11:31 UTC (from 16:31 to 19:31 LST). Meanwhile, the aerosol above $1.5 \mathrm{~km}$ has no obvious variation. According to the meteorological data, the relative humidity is $93 \% \sim 96 \%$ on 3 June. The aerosol extinction coefficient is large because of the moisture absorption effect of the aerosol particles. The extinction coefficient is retrieved above an altitude of around $400 \mathrm{~m}$ because of the loss of overlap between the laser beam and the telescope field of view.

Figure 10. The extinction coefficient profiles retrieved with different Lidar ratio.

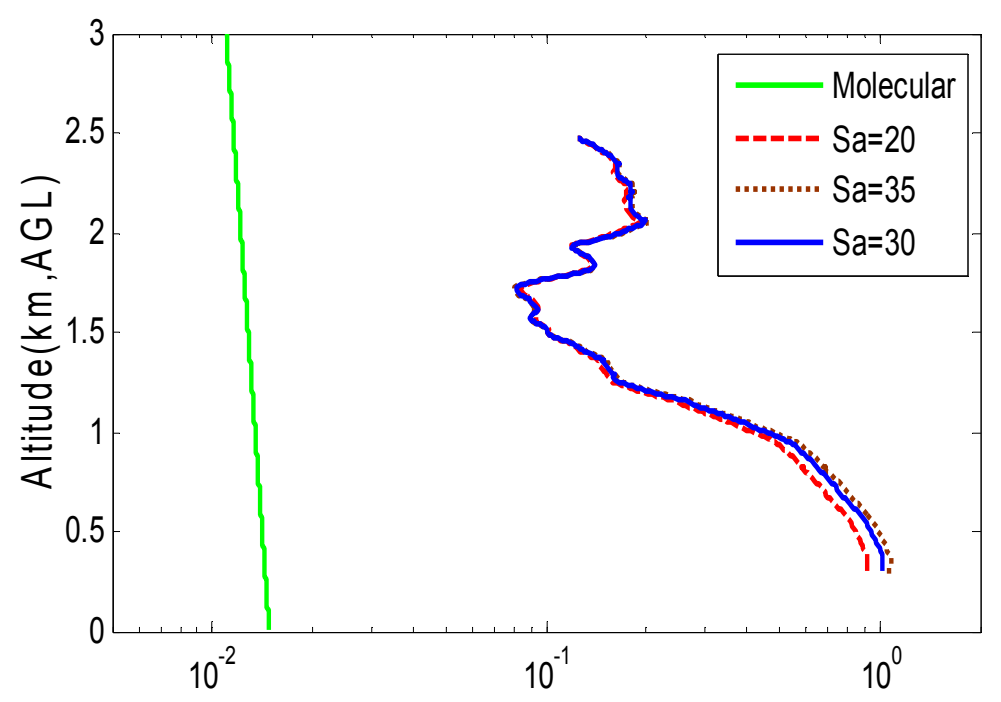

Aerosol extinction coefficient $\left(\mathrm{km}^{-1}, 20110603,12: 56\right)$ 
Figure 11. The extinction coefficient profiles on 3 June 2011, in Qingdao (data logged in LST).

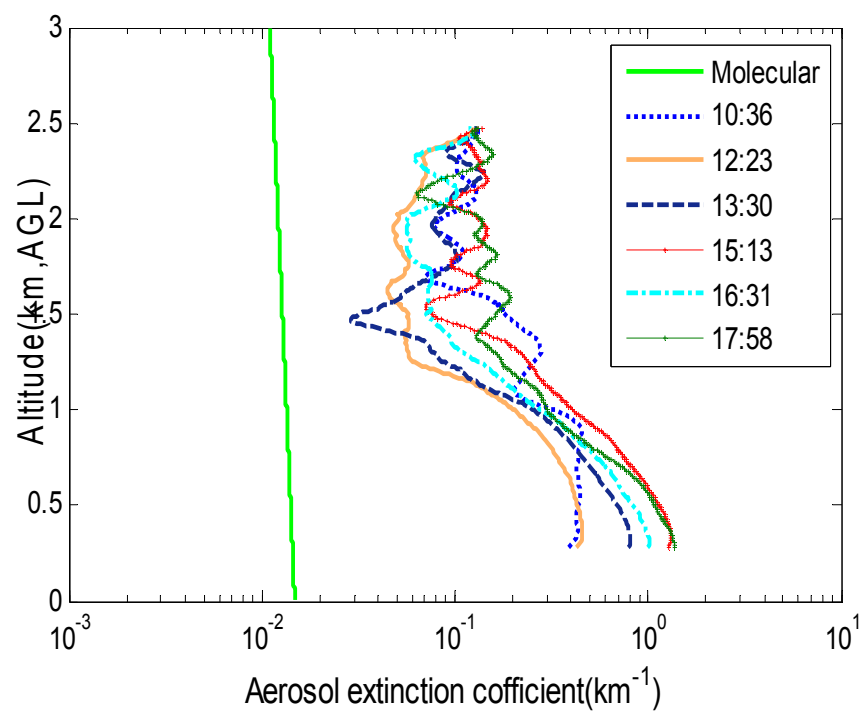

The height of the atmospheric boundary layer can be estimated using the potential temperature profile. This height is identified to be the height at which the potential temperature is subject to a steep increase. The radiosonde profiles on 3 June 2011 are plotted as Figure 12. The radiosonde was launched at approximately 8:00, 20:00 LST at the Qingdao Meteorological Station, where the longitude and latitude is $36.06^{\circ} \mathrm{N}$ and $120.33^{\circ} \mathrm{E}$ respectively. The inversion layer is obviously indicated at around $737 \mathrm{~m}$ (00:00 UTC, 08:00 LST) and $725 \mathrm{~m}$ (12:00 UTC, 20:00 LST), respectively in the radiosonde profiles. At the inversion layer, the water vapor mixing ratio at the night (12:00 UTC, 20:00 LST) is $8.63 \mathrm{~g} / \mathrm{kg}$, much higher than the mixing ratio of $4.28 \mathrm{~g} / \mathrm{kg}$ in the morning (00:00 UTC, 08:00 LST).

Figure 12. The temperature and water vapor mixing ratio profiles from the radiosonde on 3 June 2011.
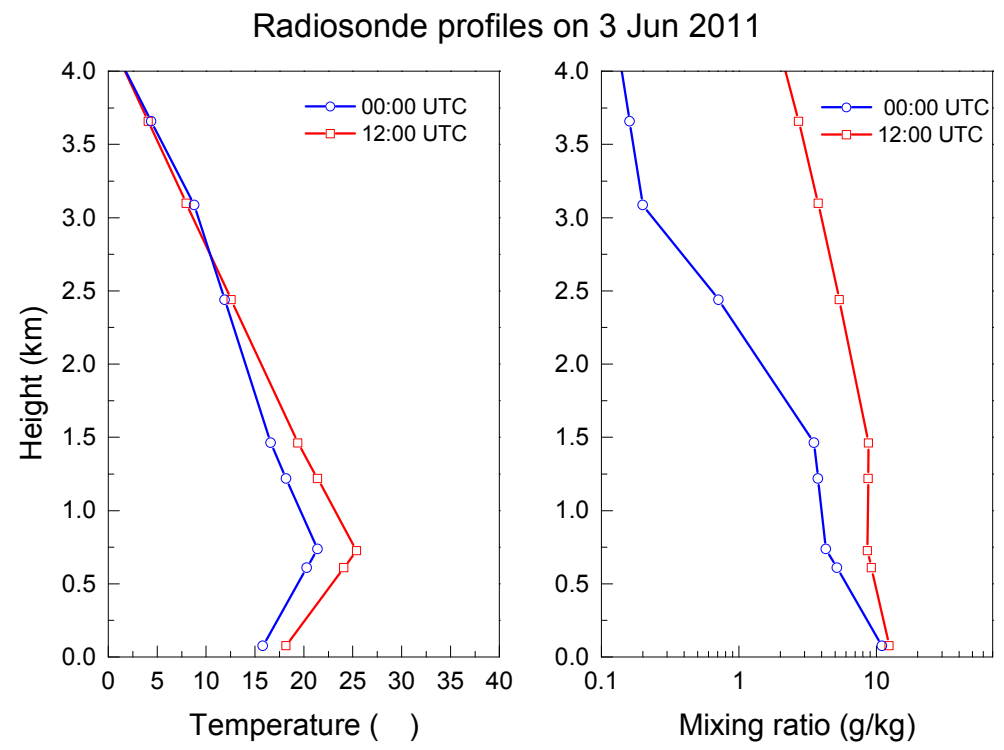
From the Fraunhofer lidar observation, the height of the boundary layer is indicated by a sharp change in the extinction profile. To investigate the development and variation of the aerosol boundary layer, Figure 13 shows the boundary layer evolution in terms of the extinction coefficient observed with the Fraunhofer lidar on 3 June 2013. The maximum detection height is around $2.48 \mathrm{~km}$ based on the SNR threshold. For this study, $30 \mathrm{~min}$ averages were used to obtain statistically meaningful boundary layer height. A stable boundary aerosol layer below approximately $1 \mathrm{~km}$ is observed. According to the local meteorological report there was a sea breeze (south-west wind) and fog emerging in the afternoon. As shown in the Figure 13, a lower layer with a height of below $800 \mathrm{~m}$ is due to the sea breeze front emerging at 15:00 LST. The boundary layer fluctuates as the front passes underneath it, showing how sea breeze propagation may produce gravity waves in the overlying stable inversion layer.

Figure 13. Temperal evolution of the extinction coefficient on 3 June 2011.

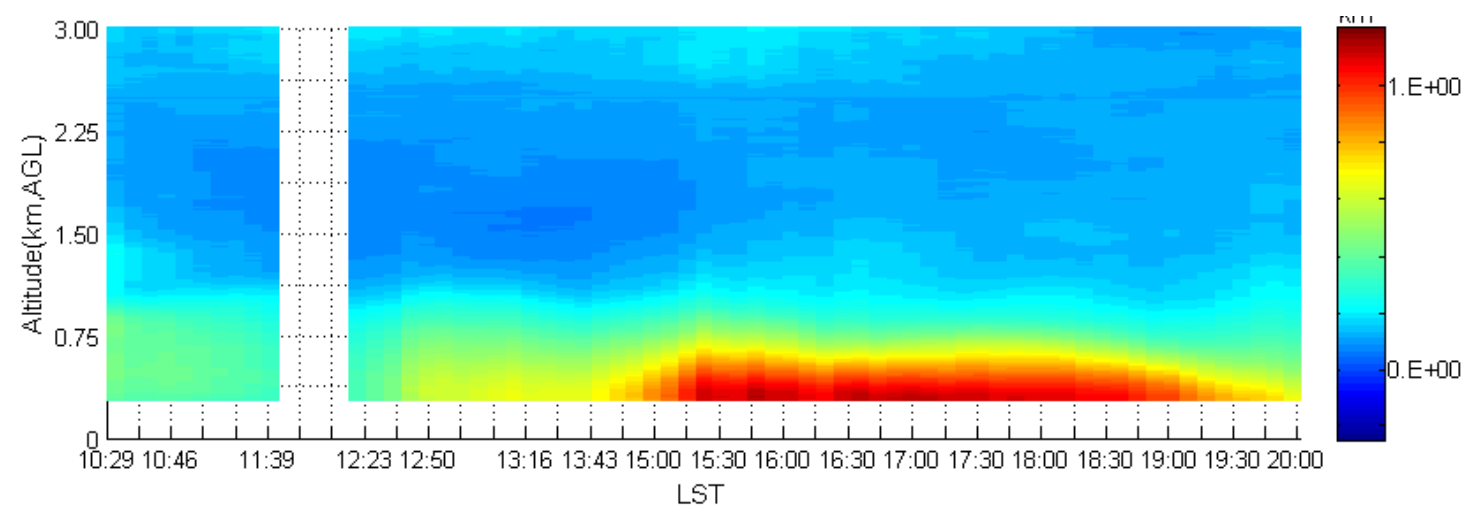

The other measurement on 24 August 2011 is observed using the Fraunhofer lidar for case study with strong variability. Figure 14 presents the vertical extinction coefficient profile on August 24, 2011. As shown in the figure, the aerosol under $1.5 \mathrm{~km}$ varies slowly while obvious variation appears when the altitude is above $1.5 \mathrm{~km}$. Moreover, the variation is almost one order of magnitude bigger because of the existence of low cloud at 03:16 UTC (11:16 LST). The radiosonde profiles are shown in Figure 15. There is not a distinct inversion layer in the temperature profile to be used to estimate the height of the boundary layer. The feature of an abrupt decrease in the water vapor mixing ratio Profile is used to mark the boundary layer around $1.5 \mathrm{~km}$. The time-height-indicator of the extinction coefficient in Figure 16 indicates that there are two layers of semi-transparent cloud occupation from 2:52 to $3: 48$ UTC (from 10:52 to 11:48 LST). The lower cloud layer top and bottom altitudes are identified as $3 \mathrm{~km}$ and $2.7 \mathrm{~km}$. The higher cloud layer top and bottom altitudes are found as $3.9 \mathrm{~km}$ and $3.6 \mathrm{~km}$. The extinction coefficient profile at 11:16 (blue plot in Figure 15) also shows a high value at the height of $3.5 \mathrm{~km}$. The high extinction and variability is obvious below $1.5 \mathrm{~km}$. The averaging time is adjusted to $15 \mathrm{~min}$ in this case due to the rapid variation.

It is important to note that daytime Fraunhofer lidar data is, unlike an ordinary Mie lidar, not noisier than that of night-time due to the absorption of the solar background by certain constituents in the solar atmosphere. From the Time-Height-Indicator figures, the aerosol varies obviously from afternoon to the dusk. Because the lidar site is in the suburb of Qingdao, the major environmental influence comes from the atmospheric turbulence rather than human activities. 
Figure 14. The extinction coefficient profiles on 24 August 2011 (data logged in LST).

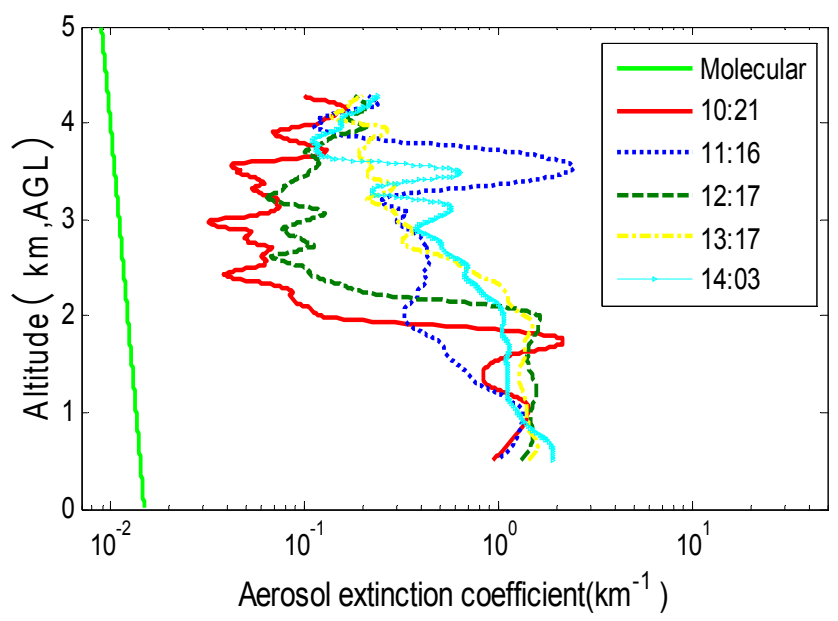

Figure 15. The temperature and water vapor mixing ratio profiles from the radiosonde on 24 August 2011.
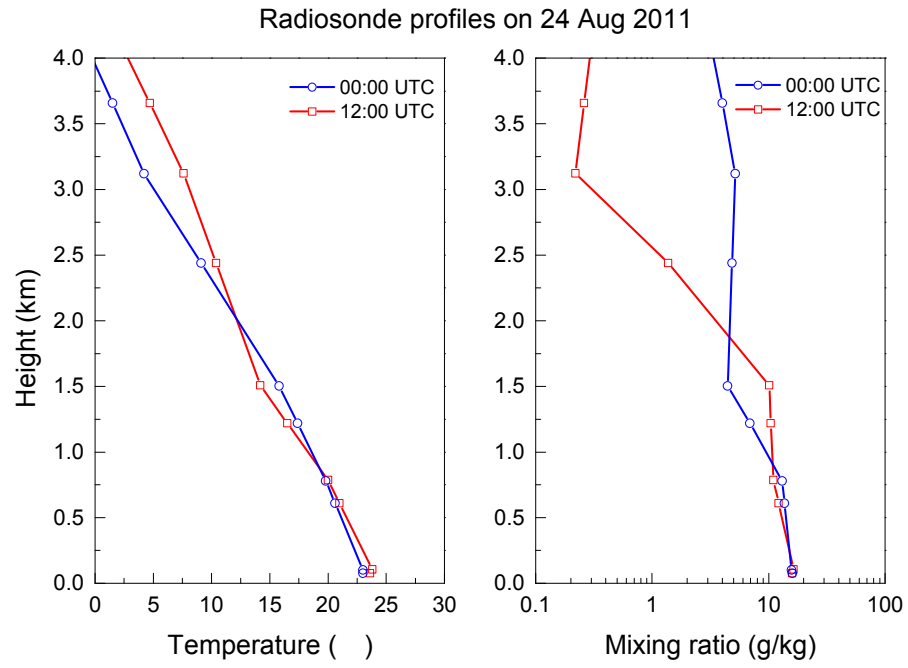

Figure 16. Temporal evolution of the extinction coefficient on 24 August 2011.

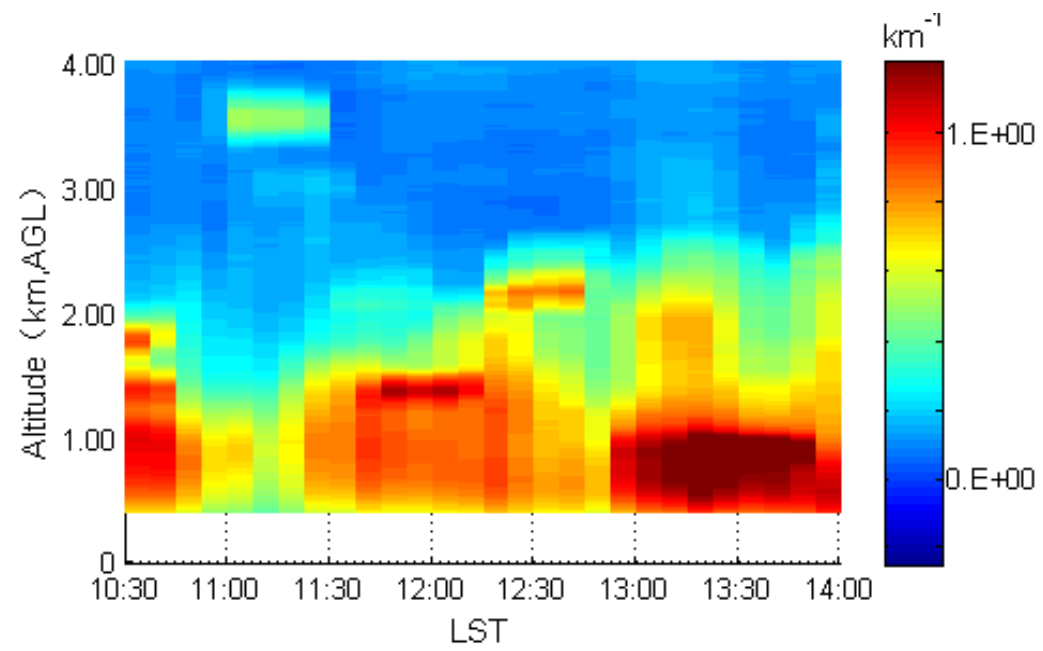


Figure 17. Comparison between the extinction coefficient of Lidar and CALIPSO Level2 data on 3 June 2011, in Qingdao.

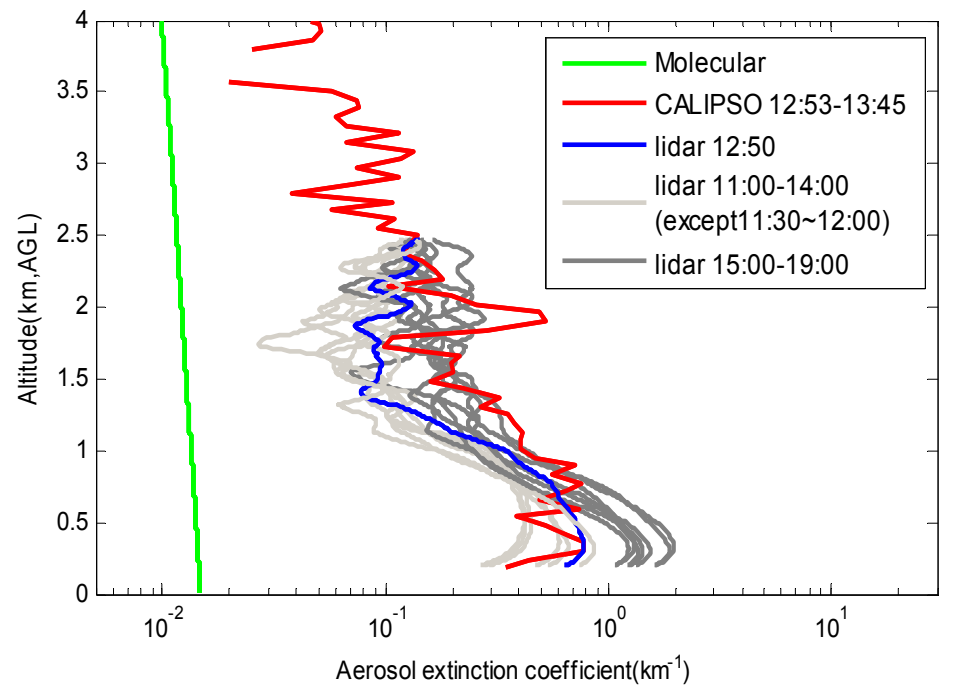

For comparison, Figure 17 presents the profiles of the aerosol extinction coefficient detected by the Fraunhofer lidar and the CALIOP level-2 aerosol profile product under cloud-free conditions (3 June 2011) with a horizontal resolution of $40 \mathrm{~km}$ and vertical resolution of $120 \mathrm{~m}$ (backscatter, extinction, and depolarization ratio). CALIOP (Cloud-Aerosol LIdar with Orthogonal Polarization) is a two-wavelength polarization-sensitive lidar onboard the NASA/CNES CALIPSO satellite (Cloud-Aerosol Lidar and Infrared Pathfinder Satellite Observation), to provide high-resolution vertical profiles of aerosols and clouds as well as on their optical properties at two wavelengths (532 $\mathrm{nm}$ and 1,064 $\mathrm{nm}$ ) [29]. The Fraunhofer lidar performed measurements before and after the CALIPSO northward-flying over the lidar location within a maximum distance of $46 \mathrm{~km}$ at 04:53 UTC (12:53 LST). The averaged CALIOP aerosol extinction profiles between 04:53 and 05:45 UTC (between 12:53 and 13:45 LST) are used for comparison. Spatial and temporal variability of aerosol results in the complex validation analysis of the Fraunhofer lidar measurement by direct comparison with the CALIOP. In this case, although CALIOP overpasses the ground based lidar location with a distance of $46 \mathrm{~km}$, the comparison illustrates that both lidars show consistent trend in the upper part of the $\mathrm{ABL}(0.7 \sim 1.5 \mathrm{~km})$ considering the spatial inhomogeneity of aerosol distributions.

\section{Conclusion}

The goal of this work is to measure the lidar backscattered signal in the green spectral region where the solar background is intensively attenuated due to the Fraunhofer absorption lines, making daytime photon counting technique possible. This is the first time, to our knowledge, that Fraunhofer lines are used for planetary boundary layer lidar remote sensing. The prototype lidar exhibits the advantage of not having to use complex noise filtering procedures, representing a viable option for an operational lidar designed to measure the atmosphere during daytime and night. The experiment detects the atmospheric parameters and characteristics of the atmospheric boundary layer. The analysis illustrates that the SNR was improved 2-3 times by operating the lidar at the wavelength in solar dark 
lines. However, the stability of the prototype is to be improved because of technical limitations. The OPO laser was the only accessible laser source at the experimental time, which represents a suitable solution for prototyping, but cannot be used for accurate and effective measurements for PBL. The advantage of using an OPO laser is its tunability to cover selected Fraunhofer lines in one lidar system. But, the OPO laser needs to achieve better frequency stability so that the frequency drift can be inhibited and the detector can accurately match with the receiving bandwidth. But, the disadvantages of using an OPO laser are low efficiency and poor beam quality. The OPO efficiency and other issues in the laser system will probably lower the outgoing laser energy by a factor of 2-3, compared to transmitting the $532 \mathrm{~nm}$ wavelength directly. Those efficiency losses of the OPO laser counteract the benefits of the SNR improvement by tuning the laser to the Fraunhofer lines. Therefore, more efficient laser transmitter should be designed for experiments.

The diagram in the dashed rectangle in Figure 2 shows the ongoing investigation to assess high spectral resolution filter coincide with wavelengths of candidate notable Fraunhofer lines, such that it is possible to isolate the signal from the solar noise and take advantage of traditional HSRL for wind, temperature and aerosol probing. There are a large number of absorption lines of iodine around solar Fraunhofer line by Magnesium at $516.4521 \mathrm{~nm}, 517.0414 \mathrm{~nm}$ and $518.2386 \mathrm{~nm}$. The idea and primary experiments in this paper present a possible solution to the operational observation problems normally associated with photon counting lidar for green wavelengths, and guarantee further investigations on lidar systems.

\section{Acknowledgments}

This work was supported by the National High-tech R\&D Program of China (Grant No. 2008AA12Z101).

\section{Conflicts of Interest}

The authors declare no conflict of interest.

\section{References}

1. Cracknell, A.P.; Hayes, L. Introduction to Remote Sensing, 2nd ed.; Taylor and Francis: London, UK, 2007.

2. Bodhaine, B.A.; Norman, B.W.; Ellsworth, G.D.; James, R.S. On Rayleigh optical depth calculations. J. Atmos. Ocean. Technol. 1999, 16, 1854-1861.

3. Donovan, D.P.; Whiteway, J.A.; Carswell, A.I. Correction for nonlinear photon-counting effects in lidar systems. Appl. Opt. 1993, 32, 6742-6753.

4. Spinhirne, J.D. Micro pulse lidar. IEEE Trans. Geosci. Remote Sens. 1993, 31, 48-55.

5. Gentry, B.M.; Chen, H.; Li, S.X. Wind measurements with 355-nm molecular Doppler lidar. Opt. Lett. 2000, 25, 1231-1233.

6. Liu, Z.S.; Liu, B.Y.; Wu, S.H.; Li, Z.G.; Wang, Z.J. A high spatial and temporal resolution mobile incoherent Doppler lidar for sea surface wind measurements. Opt. Lett. 2008, 33, 1485-1487. 
7. Liu, Z.S.; Liu, B.Y.; Li, Z.-G.; Yan, Z.-A.; Wu, S.H.; Sun, Z.-B. Wind measurements with incoherent Doppler lidar based on iodine filters at night and day. Appl. Phys. B 2007, 88, 327-335.

8. Ansmann, A.; Riebesell, M.; Wandinger, U.; Weitkamp, C.; Voss, E.; Lahmann, W.; Michaelis, W. Combined Raman elastic-backscatter lidar for vertical profiling of moisture, aerosol extinction, backscatter, and lidar ratio. Appl. Phys. B 1992, 55, 18-28.

9. Eloranta, E.E. Lidar: Range-Resolved Optical Remote Sensing of the Atmosphere. High Spectral Resolution Lidar; Springer: Berlin, Germany, 2005; pp. 143-163.

10. Hair, J.W.; Caldwell, L.M.; Krueger, D.A.; She, C.Y. High-spectral-resolution lidar with iodine-vapor filters measurement of atmospheric-state and aerosol profiles. Appl. Opt. 2001, 40, 5280-5294.

11. Browell, E.V.; Ismail, S.; Grant, W.B. Differential absorption lidar (DIAL) measurements from air and space. Appl. Phys. B 1998, 67, 399-410.

12. The Solar Spectrum. Available online: http://www.harmsy.freeuk.com/fraunhofer.html (accessed on 1 June 2010).

13. Chance, K.; Kurucz, R.L. An improved high-resolution solar reference spectrum for Earth's atmosphere measurements in the ultraviolet, visible, and near infrared. J. Quant. Spectrosc. Radiat. Transf. 2010, 111, 1289-1295.

14. Kerr, E.L. Architectural design of a ground-based deep-space optical reception antenna. Proc. SPIE 1989, doi: 10.1117/12.951701.

15. Rogers, D.J.; Bienfang, J.C.; Mink, A.; Hershman, B.J.; Nakassis, A.; Tang ,X.; Ma, L.; Su, D.H.; Williams, C.J.; Clark, C.W. Free-space quantum cryptography in the H-alpha Fraunhofer window. Proc. SPIE 2006, doi: 10.1117/12.680899.

16. Plascyk, J.A. The MK II Fraunhofer Line Discriminator (FLD-II) for airborne and orbital remote sensing of solar-stimulated luminescence. Opt. Eng. 1975, doi: 10.1117/12.7971842.

17. Smith, C.; Fischer, R.L.; Anderson, J. Detection of selected solar-stimulated fluorophores with a Fraunhofer line radiometer. J. Appl. Remote Sens. 2008, doi: 10.1117/1.2884783.

18. Stoll, M.P.; Laurila, T.; Cunin, B.; Gitelson, A.A.; Lichtenthaler, H.K.; Hame, T. FLEX: Fluorescence explorer-A space mission for screening vegetated areas in the Fraunhofer lines. Proc. SPIE 1999, doi: 10.1117/12.373087.

19. Bowman, M.R.; Gibson, A.J.; Sandford, M.C.W. Atmospheric sodium measured by a tuned laser radar. Nature 1969, 221, 456-457.

20. Zhao, Y.; Liu, A.; Gardner, C.S. Measurements of atmospheric stability in the mesopause region at Starfire Optical Range, NM. J. Atmos. Solar Terr. Phys. 2003, 65, 219-232.

21. Bills, R.E.; Gardner, C.S.; She, C.Y. Narrowband lidar technique for Na temperature and Doppler wind observations of the upper atmosphere. Opt. Eng. 1991, 30, 13-21.

22. Chu, X.; Papen, G.; Pan, W.; Gardner, C.S.; Gelbwachs, J. Fe Boltzmann temperature lidar: Design, error analysis, and first results from the North and South Poles. Appl. Opt. 2002, 41, 4400-4410.

23. Zahn, U.; Hoffner, J. Mesopause temperature profiling by potassium lidar. Geophys. Res. Lett. 1996, 26, 141-144. 
24. Gardner, C.S. Performance capabilities of middle-atmosphere temperature lidars: Comparison of $\mathrm{Na}, \mathrm{Fe}, \mathrm{K}, \mathrm{Ca}, \mathrm{Ca}+$, and Rayleigh systems. Appl. Opt. 2004, 43, 4941-4956.

25. Granier, C.; Jegou, J.P.; Megie, G. Atomic and ionic calcium in the Earth's upper atmosphere. J. Geophys. Res. 1989, 94, 9917-9924.

26. Femald, F.G. Analysis of atmospheric lidar observations: Some comments. Appl. Opt. 1984, 23, 652-653.

27. Franke, K.; Ansmann, A.; Müller, D.; Althausen, D.; Wagner, F.; Scheele, R. One year observations of particle lidar ratio over the tropical Indian Ocean with Raman lidar. Geophys. Res. Lett. 2001, $28,4559-4562$.

28. Chazette, P. The monsoon aerosol extinction properties at Goa during INDOEX as measured with lidar. J. Geophys. Res. 2003, doi: 10.1029/2002JD002074.

29. CALIPSO (Cloud-Aerosol Lidar and Infrared Pathfinder Satellite Observations). Available online: http://www-calipso.larc.nasa.gov/ (accessed on 1 June 2013).

(C) 2013 by the authors; licensee MDPI, Basel, Switzerland. This article is an open access article distributed under the terms and conditions of the Creative Commons Attribution license (http://creativecommons.org/licenses/by/3.0/). 